\title{
Energieeffiziente und drahtlose Übertragung von Sensordaten am Beispiel RFID und Bluetooth 4.0
}

\author{
Schwerpunkt: B6 - Funksensorik und Funknetzwerke \\ Simon Harasty, Christian Arnold
}

B.Eng. Simon Harasty, Wissenschaftlicher Mitarbeiter

Hochschule Fulda FB Elektrotechnik und Informationstechnik, Marquardstr. 35, 36039 Fulda

Tel.: 06619640 564, Fax: 06619640 559, email: Simon.Harasty@et.hs-fulda.de

Dipl.-Ing. (FH) Christian Arnold, Wissenschaftlicher Mitarbeiter

Hochschule Fulda FB Elektrotechnik und Informationstechnik, Marquardstr. 35, 36039 Fulda

Tel.: 06619640 557, Fax: 06619640 559, email: Christian.Arnold@et.hs-fulda.de

\section{Problemstellung}

Die drahtlose Datenübertragung findet immer mehr Verwendung in dem Aufgabenbereich der Erfassung und Übertragung von Messdaten. Dabei ist die Entscheidung für ein geeignetes Systems für eine spezielle Applikation durch die Vielzahl von Angeboten mit sehr unterschiedlichen Eigenschaften nicht trivial.

Insbesondere bei Langzeitmessungen mit batteriegestützten Systemen ist die Auswahl einer passenden Übertragung besonders wichtig, um eine optimale Betriebsdauer mit geringen Wartungsintervallen zu ermöglichen. Ohne eine Abgrenzung der applikationsspezifischen Eigenschaften ist es nur schwer möglich eine sinnvolle Entscheidung zu treffen, so dass alternative Systeme immer im Bezug auf deren Anwendungsumfeld betrachtet und verglichen werden.

In Abschnitt 5 wird die zyklische Datenerfassung von unterschiedlich positionierten Messpunkten betrachtet. Dies repräsentiert das typische Anwendungsgebiet von Funksensornetzwerken und regt einen Vergleich zwischen diesen und konventionellen Datenloggersystemen an. Auch im Feld der Funksensornetzwerke ist die Bandbreite an Systemlösungen nur schwer überschaubar. Die Schaffung eines Auswahlkriteriums erfolgt im ersten Schritt durch die Festlegung von batteriegestützten Systemen, da eine permanente Energieversorgung in vielen Anwendungsfällen nicht zur Verfügung steht und somit die Wahl der Messstellen innerhalb einer Applikation stark einschränkt.

Bei batteriegestützten Systemen sind vor allem die Konfigurationen als Funksensornetzwerk sowie als Loggersystem zu nennen. Funksensornetzwerke übergeben die gesammelten Messdaten in zyklischen Intervallen an eine Basisstation, in welcher eine Überarbeitung sowie eine Ablage der Daten erfolgt. Loggersysteme beinhalten selbst einen größeren Datenspeicher und legen die zyklisch gesammelten Messdaten intern in diesem ab, um diese später als Gesamtpaket zu übergeben. Welche dieser beiden Technologien im Bezug auf die jeweilige Applikation sinnvoll ist, kann bereits durch die Notwendigkeit der aktuellen Datenbetrachtung getroffen werden. Sollen lediglich Systemeigenschaften durch offlineMessungen analysiert werden, so ist ein Loggersystem ausreichend. Zur Realisierung echtzeitfähiger Aufgaben, wie etwa der Integartion in Steuerungen und Regelungen, muss allerdings ein Zugang zu Online-Messdaten geschaffen werden, so dass lediglich die zyklisch übertragenden Funksensornetzwerke eingesetzt werden können. Eine vergleichende Untersuchung des Energiehaushaltes wird in Abschnitt 4 vorgenommen.

Im Folgenden werden verschiedene Systeme und Konfigurationen untersucht. Das Hauptaugenmerk liegt auf dem Vergleich der Übertragungstechnologien Bluetooth 4.0 und RFID (Abschnitt 3), in Anwendung des Systems FlexLog (Abschnitt 2). Die fokussierten Applikationen umfassen Langzeitmessungen in historischen Gebäuden, welche meist aufgrund des Denkmalschutzes nicht über die Installation einer elektrischen Energieversorgung und einer drahtgebundenen Kommunikationsinfrastruktur verfügen.

\section{System FlexLog}

Die Erfassung individueller Messgrößen erfordert meist die Investition in geeignete Messsysteme oder einen entsprechenden Aufwand zur Anpassung bestehender Universalsysteme. Daher werden Messsysteme mit problemspezifischen Lösungen, sogar bei teilweise sehr ähnlichen Anforderungen der 
Messdatenerfassung, genutzt. Aufgrund der wechselnden Anforderungen, Umgebungseinflüsse und Applikationen ist das Portfolio marktverfügbarer Produkte entsprechend groß.

Um eine universelle Messplattform zur Verfügung zu stellen, wurde das flexibles System „FlexLog“ entwickelt.

Hierbei handelt sich um einen modular aufgebauten Datenlogger mit frei konfigurierbaren Schnittstellen zu Sensorik, Datenübertragung und Energieversorgung (Abbildung 1), damit das System an die jeweiligen Anforderungen schnell angepasst und konfiguriert werden kann.

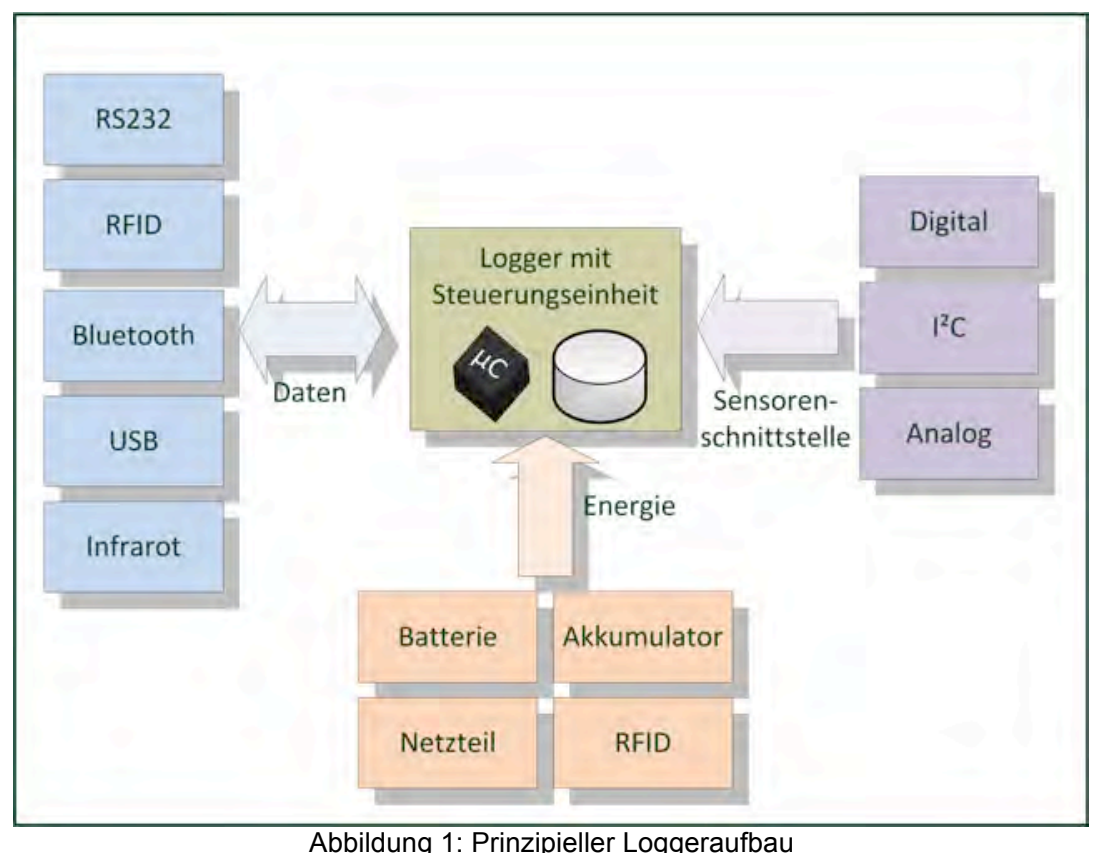

Als Schnittstellen zu Sensorsystemen werden vier analoge Kanäle (10-Bit Strom, Spannung und Widerstandsbestimmung nach entsprechender Konfiguration), eine digitale Zustandserfassung und eine $I^{2} \mathrm{C}$-Schnittstelle bereitgestellt. An die $\mathrm{I}^{2} \mathrm{C}$-Schnittstelle können, wie für diesen Bus üblich, mehrere Teilnehmer angeschlossen werden oder bei gleicher Adressierung (herrscht bei diversen Sensoren vor) zwei Teilnehmer an getrennten Buskanälen.

Zum Auslesen der gespeicherten Daten des Loggers mit einem Rechnersystem sind sowohl kabelgebundene als auch drahtlose Lösungen realisiert. Als kabelgebundene Technologien stehen RS232 und USB zur Verfügung. Die drahtlose Datenübertragung ist über:

- RFID (für kurze Strecken mit geringem bis hin zu gar keinem Energieverbrauch, da die für die Übertragung benötigte Energie aus dem elektromagnetischen Feld des RFID-Lesegeräts bereitgestellt wird) [FIN08]

- Bluetooth 4.0 (für eine höhere Reichweite) [SIG12] und möglich.

- Infrarot (als Referenz zum Vergleich)

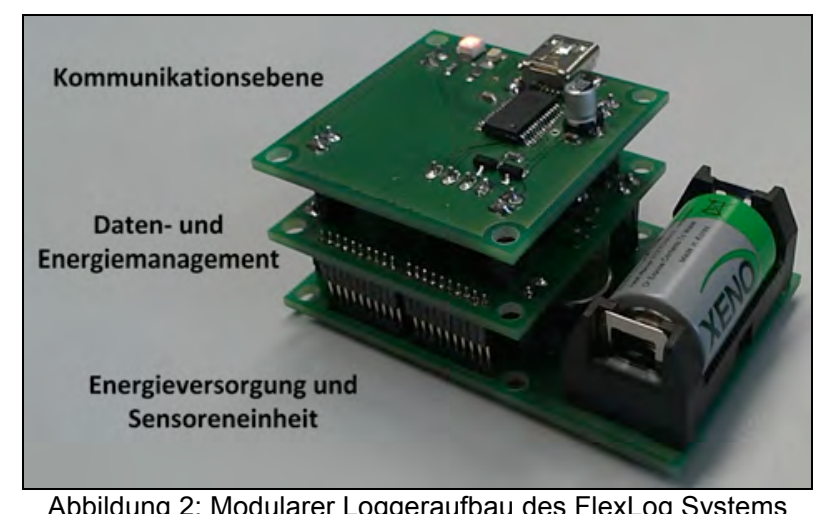


Die Energieversorgung kann ebenfalls über vier verschiedene Varianten realisiert werden. Eine einfache und günstige Lösung bieten Batterien, die allerdings in festen Intervallen gewechselt werden müssen. Eine dauerhafte Versorgung wird über ein Netzteil realisiert. Als Mischlösung steht ein Akkumulator mit Lademanagement zur Verfügung. Zusätzlich wurde die Möglichkeit der drahtlosen Energieversorgung über die Kommunikation via RFID integriert. Für Energy Harvesting-Lösungen steht eine universelle Schnittstelle zu Akkumulator und Mikorcontroller (für Lademanagement) bereit. Die Realisierung des Konzeptes erfolgte in einem modularen Aufbau (siehe Abbildung 2) mit vertikal steckbaren Loggermodulen.

Das Herzstück stellt hierbei das mittlere Modul durch die Realisierung des Energie- und Datenmanagement dar. Verwendete Komponenten werden über dieses Management je nach Bedarf zuund abgeschaltet, Messdaten werden erfasst und im Speicher abgelegt. Dieses Modul ist in sämtlichen Systemkonfigurationen gleich.

Die Kommunikationsebene kann mit dem jeweils gewünschten Modul wie RFID, Bluetooth, Infrarot oder wie hier abgebildet USB ausgestattet werden. Ebenfalls ist das Sensor und Energiemodul je nach Systemkonfiguration austauschbar. Dieses Modul umfasst Anpassungsschaltungen zur Sensorik, die Ausführung der Schnittstellen und die jeweilige Energieversorgung.

\section{Vergleich drahtloser Schnittstellen}

Für eine Gegenüberstellung der verschiedenen drahtlosen Schnittstellen zur Verwendung an einem Datenloggersystem, werden zunächst die Grundlagen der einzelnen Technologien beschrieben und anschließend miteinander verglichen. Die verwendeten Datenübertragungstechnologien sind prinzipiell sehr ähnlich, finden jedoch in verschiedenen Anwendungsumgebungen Verwendung. Um eine bessere Positionierung der Systeme untereinander zu Gewährleisten wird ebenfalls die Infrarotübertragung hier betrachtet.

\subsection{Funkkommunikation über weite Strecken am Beispiel Bluetooth 4.0}

Für eine einfache Anbindung von Peripheriegeräten über eine drahtlose Schnittstelle wurde das auf dem IEEE 802.15.1 [IEE02] Standard basierende Bluetooth über einen Zusammenschluss der Industrie (Bluetooth Special Interrest Group; SIG [SIG12]) entwickelt. Bei einem Bluetoothsystem wird die Datenübertragung im Frequenzband von $2,4 \mathrm{Ghz}$ (ISM) realisiert, welches ebenfalls von anderen Funktechnologien wie WLAN oder ZigBee genutzt wird. Zur Vermeidung von Störungen wurde Bluetooth, anstatt auf einer festen Frequenz, über eine nach jedem Paket wechselnde Frequenz realisiert. Dieses Verfahren wird als Frequenzy Hopping bezeichnet und nutzt bei Bluetooth 79 Kanäle mit jeweils einer Bandbreite von $1 \mathrm{MHz}$. [GES09]

Die aktuelle Version 4.0 soll die Anbindung verschiedenster Geräte noch effizienter und energiesparender ermöglichen, wodurch eine ähnliche Charakteristik zu Konkurrenzsystemen wie ZigBee erreicht wird. Die Bluetooth SIG verabschiedete im Dezember 2009 den neuen Standard mit dem erweiterten Protokollstapel „Low Energy“ [SIG12], welcher eine energieeffizientere Übertragung ermöglicht. Der in dieser Veröffentlichung betrachtete Bluetooth Chip [BLU11] bietet bei einer Sendeleistung von 0dBm eine Stromaufnahme von lediglich $27 \mathrm{~mA}$ und im Sleepmodus lediglich $400 \mathrm{nA}$. Diese Werte liegen unter denen früher Bluetooth Konfigurationen und erlauben somit einen batteriegestützten Betrieb über einen längeren Zeitraum. Allerdings ist zu bedenken, dass diese Sendeleistung lediglich für Übertragungsreichweiten von bis zu 40m im Gegensatz zum Maximum bei einer Sendeleistung von $+4 \mathrm{dbm}$ mit 150m liegt. Eine Übersicht über Sendeleistung Stromaufnahme und Reichweite ist in Tabelle 1 dargestellt. Ebenfalls ist das System skalierbar, wodurch die Sendeleistung an entsprechende Entfernungen angepasst und hierdurch der Energiehaushalt optimiert oder die Reichweite erhöht werden kann.

Bei der Verwendung des Low Energy Protokollstapels liegt die Datenübertragung bei 1Mbit/s und wird über Gaußsche Frequenzumtastung (GFSK; Gaussian Frequency Shift Keying; Gaußfilter geglättete Frequenzumtastung) moduliert. [GES09]

\begin{tabular}{|c|c|c|}
\hline Sendeleistung & Reichweite & Stromaufnahme \\
\hline$+4 \mathrm{dBm}$ & $\sim 150 \mathrm{~m}$ & $32 \mathrm{~mA}$ \\
\hline$+0 \mathrm{dBm}$ & $\sim 40 \mathrm{~m}$ & $27 \mathrm{~mA}$ \\
\hline$-23 \mathrm{dBm}$ & $\sim 10 \mathrm{~m}$ & $21 \mathrm{~mA}$ \\
\hline \multicolumn{2}{|c|}{ Tabelle 1: Energiebedarf und Reichweite im Sendebetrieb des BLE112 [BLU11] }
\end{tabular}

\subsection{Funkkommunikation über kurze Strecken am Beispiel RFID}


Zurzeit wird RFID in vier unterschiedlichen Frequenzbereichen genutzt: LF = Low Frequency 125kHz; HF $=$ High Frequency 13,56MHz; UHF = Ultra High Frequency $800 \mathrm{MHz}$; und MW = Microwave 2,45GHz. Die unterschiedlichen Frequenzen gehen einher mit den verschiedenen Übertragungsgeschwindigkeiten wie auch mit Bauteilgrößen und Bauteilkosten. Ebenfalls ist die Kopplung der einzelnen Systemkomponenten über den Frequenzbereich festgelegt: LF und HF Systeme verfügen über eine magnetische Kopplung.

Lesegerät und Transponder müssen sich in einem nahen Bereich befinden um diese Kopplung zu gewährleisten. Die Begrenzung dieses Bereichs ist durch den Übergang des ausgesendeten Feldes des Lesegeräts in den elektro-magnetischen Bereich limitiert. UHF und MW Systeme sind elektromagnetisch gekoppelt und senden bzw. empfangen entsprechende elektromagnetische Wellen. Dies ermöglicht höhere Übertragungsreichweiten, gerade bei gerichteten Systemen. Eine Übersicht der Eigenschaften der unterschiedlichen Frequenzbereiche ist in Tabelle 2 aufgeführt. [Fin08]

\begin{tabular}{|c|c|c|c|c|}
\cline { 2 - 5 } \multicolumn{1}{c|}{} & LF & HF & UHF & MW \\
\hline $\begin{array}{c}\text { Typische } \\
\text { Frequenz }\end{array}$ & $125 \mathrm{kHz}$ & $13,56 \mathrm{MHz}$ & $34,45 \mathrm{GHz}$ \\
\hline Wellenlänge & $2,4 \mathrm{~km}$ & $22,1 \mathrm{~m}$ & $3 \mathrm{~m}$ & $12,2 \mathrm{~cm}$ \\
\hline $\begin{array}{c}\text { Maximale } \\
\text { Reichweite passiv }\end{array}$ & $30 \mathrm{~cm}$ & $1-1,5 \mathrm{~m}$ & $2 \mathrm{~m}$ \\
\hline Kopplungsart & induktiv & induktiv & elektromagnetisch & elektromagnetisch \\
\hline
\end{tabular}

Tabelle 2: Eigenschaften von RFID Systemen [OPA11, FIN08]

Die Integration eines Mikrocontrollers kann am einfachsten mit einem LF- oder HF-System geschehen. $\mathrm{Da}$ mit der höheren Frequenz eines HF Systems $(13,56 \mathrm{MHz})$ kleinere Spulenbauformen als bei LF Systemen erreicht werden können, wurde dieses System als am besten geeignet für diese Applikation befunden. Ein weiteres Unterscheidungsmerkmal für RFID Systeme ist die Energieübertragung vom Lesegerät zu den jeweiligen Messstellen (Transpondern) und umgekehrt. Es wird zwischen passiven, semipassiven und aktiven Systemen unterschieden (vgl. [HAR1]), wobei sich diese Angaben auf die zum Betrieb benötigte unterstützende Energie bezogen wird. Passive RFID-Transponder erhalten die gesamte zur Nutzung benötigte Energie über das magnetische Feld des Lesegeräts und können so vollkommen ohne unterstützende Energiequelle betrieben werden. Allerdings muss zur Messung das Lesegerät stets in der Nähe des Transponders sein (vgl. [COS11], [HAR3]). Die Energie für die Kommunikation wird bei semipassiven Transpondern vollständig vom Lesegerät zur Verfügung gestellt, wodurch eine erhöhte Kommunikationsreichweite erreicht werden kann. Die digitalen Komponenten des Transponders werden durch ein unterstützendes System mit Energie versorgt. Aktive Transponder entsprechen, nach ihrer komplett energiegestützten Kommunikation und Steuerung eher der Spezifikation eines Telemetriesenders als einem RFID Transponders. Allerdings bieten hierdurch aktive Transponder die höchste Kommunikationsreichweite. Die drei Konfigurationen sind in Abbildung 3 skizziert. [FIN08]

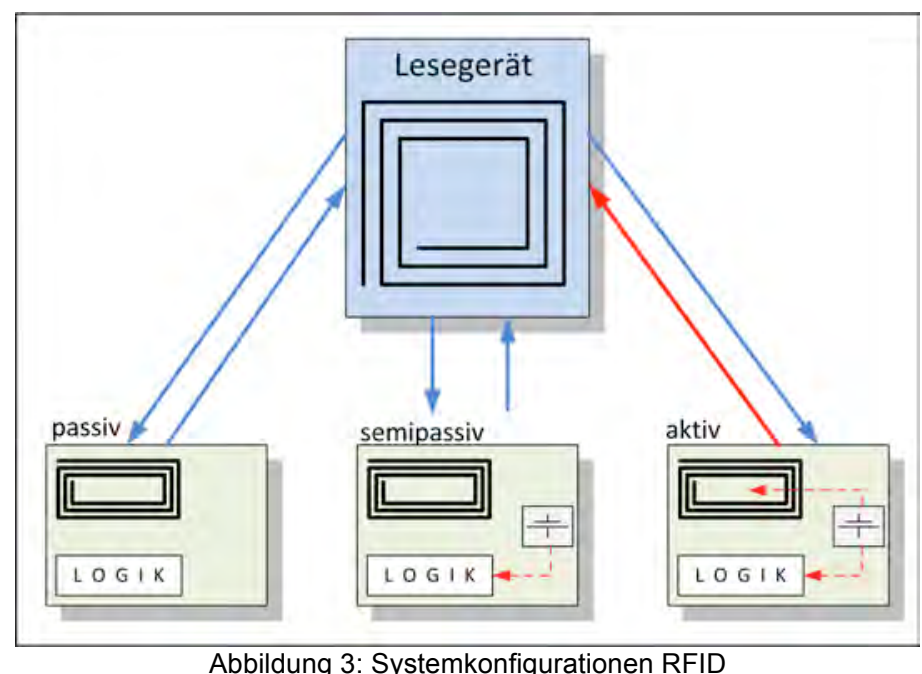

Typische Normen für den HF Frequenzbereich eines RFID-Systems sind ISO 15693 [ISO15] und ISO 14443 [ISO14], welche die Kopplung von Nahfeld RFID-Systemen beschreiben. Diese Normen beziehen sich allerdings auf das Hauptanwendungsgebiet der Identifikation, welche hier eine eher untergeordnete Rolle spielt. Eine triviale Nutzung zur Anbindung kann über EEPROM-Speicherbausteine mit RFID- und 
digitaler Schnittstelle erfolgen, da das Lesen und Schreiben solcher Speicher bereits in aktueller Normung vorgesehen ist. Diese Anbindung bzw. deren Nutzung zur bidirektionalen Kommunikation ist durch die limitierten Schreibzyklen eines EEPROMS stark eingeschränkt. EEPROM Bausteine verfügen typischerweise über ca. 100.000 Schreibzyklen, welche bei einer bidirektionalen Kommunikation mit laufender Paketbestätigung eine starke Einschränkung des Nutzungszeitraums darstellen. Aufgrund dieser Tatsachen wurde ein alternativer Ansatz gewählt.

Zur Anbindung des Datenloggers wurde eine eigene RFID-Kommunikationsstruktur entworfen. Diese ermöglicht eine schnellere Übertragung und Nutzung der Schnittstelle mit direkter Anbindung an einem Mikrocontroller. Die Übertragungsrate variiert je nach Konfiguration. Eine für diese Systemart typische Angabe ist $100 \mathrm{kbit} / \mathrm{s}$.

\subsection{Optische Kommunikation über kurze Strecken am Beispiel Infrarot als Alternative}

Als dritte drahtlose Kommunikationsschnittstelle wird die Infrarotübertragung betrachtet. Seit 1993 wird mit der Infrared Data Association (IrDA) darauf gezielt eine genormte Datenübertragung im Infrarotbereich zu erreichen. Die daraus resultierende und günstigste Lösung zur Datenübertragung ist das Serial Infrared (SIR). SIR bietet die gleichen Datenraten wie die an Mikrocontrollern übliche UARTSchnittstelle und deren Ursprung, nämlich die RS-232 Schnittstelle. Typische Übertragungsraten liegen hier zwischen $9,6 \mathrm{kbit} / \mathrm{s}$ bis hin zu 115kbit/s. Bei der Infrarot Übertragung wird ein einfaches gepulstes Lichtsignal benutzt, welches sich außerhalb des für den Menschen sichtbaren Spektrums befindet. Je nach verwendetem Transceiver können die Übertragungsreichweiten stark variieren. Ein für einen Anwendungsfall mit einer Reichweite von $20-30 \mathrm{~cm}$ geeignetes System benötigt für die Erstellung eines Pulses eines Signals ca. 500mA (TFBS4650 [VIS09]). Liegt allerdings die Pulsrate bei lediglich $20 \%$ (Puls: $20 \%$ eingeschaltet, $80 \%$ ausgeschaltet), so kann ein mittlerer Strom von 100mA angenommen werden. Bei einer Übertragung hoher Datenmengen ist eine direkte Sichtverbindung zwischen den zwei kommunizierenden Geräten zwingend erforderlich. [KNU04]

\section{Energetischer Vergleich von Sensornetzwerk, Datenlogger oder Hybrid}

Die Auswahl eines jeweils für die Applikation passenden Datenübertragungssystems hängt in erster Linie von der Verwendung der erfassten Daten, der Umgebung sowie den verfügbaren finanziellen Mittel ab. Um diese Faktoren zu minimieren wird im Folgenden die im Anwendungsfall geforderte Aktualität der Daten als nicht gefordert und die Umgebung für sämtliche Systeme geeignet angenommen.

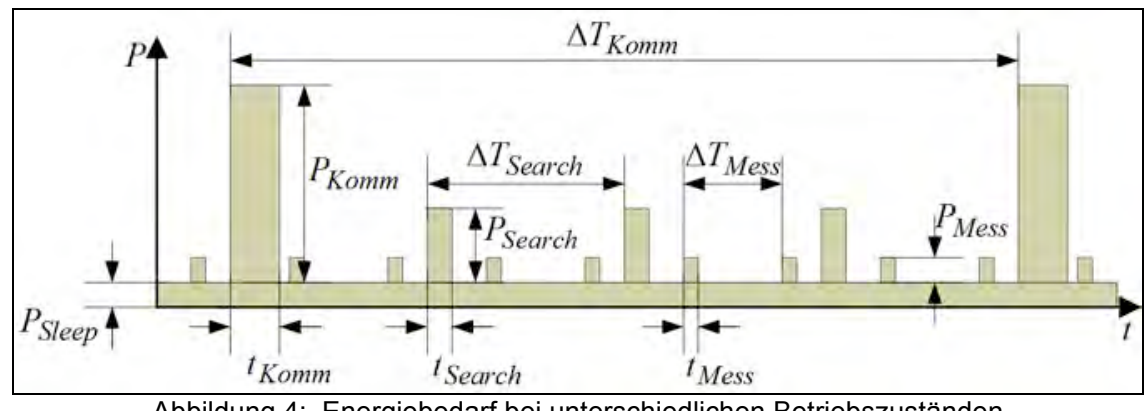

Abbildung 4: Energiebedarf bei unterschiedlichen Betriebszuständen

Um den Energiehaushalt der Messstelle abzuschätzen, muss der jeweilige Leistungsbedarf bei unterschiedlichen Betriebszuständen ermittelt werden. Die unterschiedlichen Betriebszustände sind in der Abbildung 4 dargestellt und wie folgt definiert:

- $\quad$ Sleepmodus

Weder Kommunikations- noch Sensorikmodule sind aktiv. Sämtliche Bauteile sind abgeschaltet oder befinden sich im Sleep-Modus, so dass der Grundleistungsbedarf $P_{\text {Sleep }}$ benötigt wird.

\section{- $\quad$ Messung}

Die Messstelle führt die Erfassung mit der bestückten Sensorik durch. Dies geschieht im Intervall $\Delta T_{\text {Mess }}$ mit einer Dauer von $t_{\text {Mess }}$, wobei in dieser Zeit die mittlere Leistung $P_{M e s s}$ bezogen wird.

\section{- $\quad$ Suche nach Kommunikationspartner}

Die Messstelle sucht nach einem Kommunikationspartner. Dies geschieht im Intervall $\Delta T_{\text {Search }}$ mit einer Dauer von $t_{\text {Search }}$, wobei in dieser Zeit die mittlere Leistung $P_{\text {Search }}$ bezogen wird.

- Datenübertragung 
Die Messstelle kommuniziert mit dem Partner. Dies geschieht im Intervall $\Delta T_{\text {Komm }}$ mit einer Dauer von $t_{K o m m}$, wobei in dieser Zeit die mittlere Leistung $P_{\text {Komm }}$ bezogen wird.

Durch aufsummieren der Leistungen über die Zeit kann der Energiebedarf abgeschätzt werden. Um die einzelnen Sensorsystemkonfigurationen miteinander zu vergleichen, wird die mittlere Leistung ermittelt:

$\bar{P}_{\Sigma}=P_{\text {Sleep }}+P_{\text {Mess }} \cdot \frac{t_{\text {Mess }}}{\Delta T_{\text {Mess }}}+P_{\text {Search }} \cdot \frac{t_{\text {Search }}}{\Delta T_{\text {Search }}}+P_{\text {Komm }} \cdot \frac{t_{\text {Komm }}}{\Delta T_{\text {Komm }}}$

Dabei ist zu bedenken, dass die Kommunikationsdauer von der zu übertragenden Datenmenge $D_{E}$ und somit auch vom Messintervall abhängig ist. Diese Zeit kann mit Hilfe der Übertragungsrate $v_{s}$ sowie der Kenntnis über das Datenvolumen pro Messung $D_{E}$ ermittelt werden, wobei der Bedarf für den Aufbau der Verbindung hier vernachlässigt und in den Suchmodus eingerechnet wird.

$t_{\text {Komm }}=\frac{D_{E} \cdot \Delta T_{\text {Komm }}}{v_{s} \cdot \Delta T_{\text {Mess }}}$

Einsetzen in Gleichung (1) liefert nach entsprechender Vereinfachung schließlich:

$\bar{P}_{\Sigma}=P_{\text {Sleep }}+P_{\text {Mess }} \cdot \frac{t_{\text {Mess }}}{\Delta T_{\text {Mess }}}+P_{\text {Search }} \cdot \frac{t_{\text {Search }}}{\Delta T_{\text {Search }}}+P_{\text {Komm }} \cdot \frac{D_{E}}{v_{s} \cdot \Delta T_{\text {Mess }}}$

Der Energiebedarf der Kommunikation hängt somit nur noch indirekt vom Kommunikationsintervall ab, da die mittlere Energie bezogen auf das Abtastintervall betrachtet wird. Hierdurch ist die für die Datenübertragung benötigte Energie vom Messintervall bzw. der damit verbundenen Datenmenge abhängig.

Gleichung (3) gilt im Allgemeinen für einen Funkdatenlogger, hingegen vereinfacht sich die Betrachtung beim Einsatz in einem Funk-Sensornetzwerk (ohne Hopping-Funktionen), da hier typischerweise sämtliche Intervalle gleich dem Messintervall gesetzt werden. Bei der Nutzung eines RFID-Datenloggers hingegen entfällt der Energiebedarf für die Suche nach dem Kommunikationspartner und die Kommunikation selbst, somit ist diese Technologie mit einem drahtgebundenem Datenlogger vergleichbar.

Bei der für den Einsatzbereich typische Datenerfassung im Minutenbereich gilt $P_{\text {Mess }} \cdot t_{\text {Mess }} \ll P_{\text {sleep }} \cdot t_{\text {sleep }}$, da der Zeitraum der Messung wesentlich kleiner ist und die so entstehende Leistungsspitze nicht mehr relevant ist. Hierbei gilt für $P_{\text {Base }}$ die gemessene Leistung von $264 \mu \mathrm{W}$. Sowohl bei aktivem Funkdatenlogger wie auch bei Funk-Sensornetzwerk werden jeweils die BluetoothVerbindungen mit einer Reichweite von bis zu $150 \mathrm{~m}$ mit einer Betriebsspannung von 3,3V verwendet. Tabelle 3 vergleicht die verschiedenen Technologien hinsichtlich des Energiebedarfs.

\begin{tabular}{|l|l|}
\hline System & Mittlere Leistung \\
\hline Aktiver Funk-Datenlogger & $\bar{P}_{\Sigma}=P_{\text {Base }}+P_{\text {Search }} \cdot \frac{t_{\text {Search }}}{\Delta T_{\text {Search }}}+P_{K o m m} \cdot \frac{D_{E}}{v_{s} \cdot \Delta T_{\text {Mess }}}$ \\
\hline Funk-Sensornetzwerk & $\bar{P}_{\Sigma}=P_{\text {Base }}+\frac{P_{\text {Search }} \cdot t_{\text {Search }}+P_{\text {Komm }} \cdot \frac{D_{E}}{v_{s}}}{\Delta T_{\text {Mess }}}$ \\
\hline RFID Datenlogger & $\bar{P}_{\Sigma}=P_{\text {Base }}$ \\
\hline
\end{tabular}

Tabelle 3: Energiebestimmung für aktive Funk-Datenlogger, Funk-Sensornetzwerke und RFID Datenlogger 
Um die Abhängigkeit der benötigten Leistung genauer zu betrachten ist in Abbildung 4 (rechts) die Leistung eines aktiven Datenloggers mit Bluetooth-Schnittstelle in Abhängigkeit dieser Größen dargestellt. Es werden typische Bereiche mit Messintervallzeiten zwischen 10 Sekunden und 5 Minuten sowie ein Kommunikationsintervall zwischen einer Stunde und 1 Tag betrachtet. Die Auswirkung des Messintervalls ist zwar vorhanden, wirkt sich allerdings nicht signifikant auf den Energiehaushalt des Systems aus. Dieser Effekt ist durch das vergleichsweise niedrig angesetzte, allerdings in etlichen Anwendungen wie etwa der Klimadatenmessung angemessene Datenvolumen $D_{E}$ von 120 Bit pro Messung begründet. Die Datenübertragungsrate $v_{s}$ des Bluetooth Systems ist mit $1 \mathrm{Mbit} / \mathrm{s}$ wesentlich höher, so dass die Auswirkungen der Datenmenge in Bereichen von Messintervallen über einer Minute fast keine Auswirkungen auf den Energiehaushalt des Systems nehmen.

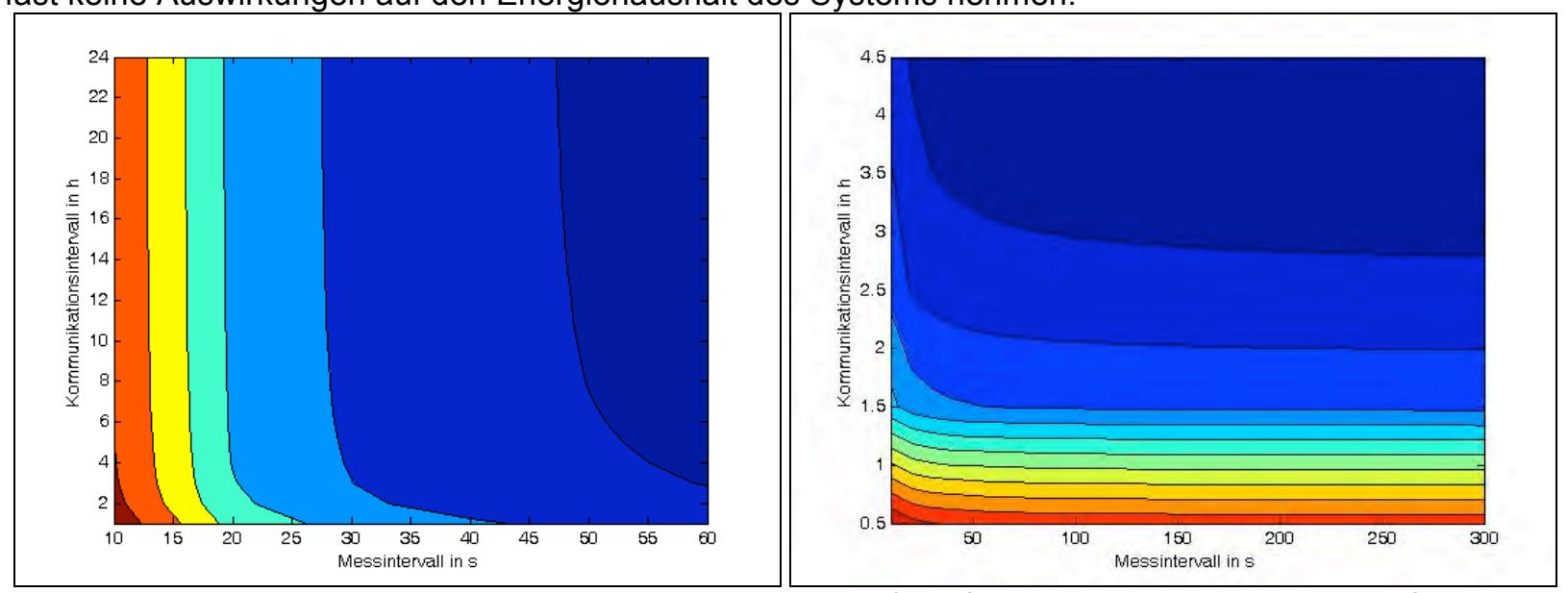

Abbildung 5: Durchschnittliche Leistung eines aktiven Datenloggers mit Infrarot-Schnittstelle (links) und mit Bluetooth Schnittstelle (rechts)

In Abbildung 5 (links) ist die Leistung des Datenloggersystems mit Infrarotschnittstelle dargestellt. Es werden die gleichen Intervalle wie bei der Bluetooth Schnittstelle betrachtet. In diesem Konfigurationsfall ist die Auswirkung des Messintervalls dominierender, da die Datenrate des Systems mit 9600 Baud rund 100 fach kleiner ist als die des Bluetooth Systems, während die Verbindungszeit rund 10mal geringer ist. Allerdings ist der Grundverbrauch des Systems durch eine sparsamere Anbindung geringer. Die konkrete Betrachtung des entwickelten Datenloggers wurde anhand der am Datenlogger gemessenen Leistungen vorgenommen.

\section{5. Ökonomischer Vergleich am Beispiel der Klimadatenerfassung}

$\mathrm{Zu}$ den Aufgaben der präventiven Konservierung zählen sämtliche Aufgaben, die die Umgebungsbedingungen von Kulturgütern derart gestalten, dass Wertminderung und Verlust verhindert oder verzögert werden (vgl. [DIN09]). Typische Gebäude sind Bibliotheken, Magazine, Archive, Depots, Museen, Kirchen oder Schlösser. Folglich ist die Gestaltung der Klimabedingungen eine Teilaufgabe der präventiven Konservierung, welche allerdings nur interdisziplinär von Restauratoren, Materialwissenschaftlern, Bauphysikern, Bauingenieuren, Klima- und Automatisierungstechnikern und anderen gelöst werden kann. Für aktive Maßnahmen in der Optimierung der Raumluft durch Anpassung des Nutzungsverhaltens und dem gezielten Einsatz von Klimatechnik ist eine Online-Erfassung unumgänglich. Zu den passiven Maßnahmen hingegen zählen Anpassungen der Gebäudefassade. Zur Durchführung einer derartigen Optimierung ist jedoch eine Analyse der aktuellen bauklimatischen Situation erforderlich, welche typischerweise über Datenlogger erfasst wird, eine Online-Datenerfassung ist nicht erforderlich. (vgl. [ARN11])

Weiterhin wird in Applikationen der präventiven Konservierung typischerweise das Klima mit Datenloggern erfasst um bauphysikalische Veränderungen sowie dessen Folgen zu identifizieren und letztendlich die Klimasituation mehr oder weniger zu kontrollieren. Diese Art der Klimakontrolle erfordert allerdings einen nicht zu unterschätzenden Personaleinsatz zum regelmäßigen Auslesen und Archivieren der Daten. Um Klimadaten für die Überwachung, Diagnose, Steuerung und Planung Online zu erfassen, ist der Einsatz eines drahtlosen Sensornetzwerkes meist unumgänglich, da die Installationen drahtgebundener Systeme einen nicht zu unterschätzenden Eingriff in die ggf. denkmalgeschützte Bausubstanz erfordert. Der Einsatz von Funksensornetzwerken gehört in diesem Bereich bereits zum Stand der Technik (vgl. [LIN08], [YON09]), wobei des Öfteren die zunächst hohe Investition in 
Funksensornetzwerke auf den Anwender abschreckend wirken und deswegen üblicherweise auf die Verwendung von Datenloggern zurückgegriffen wird.

Nach den Analysen aus Kapitel 4 wird deutlich, dass bei Verwendung moderner Kommunikationstechnologien der Einsatz einer Messstelle als Datenlogger (DL) oder im Funksensornetzwerk (Wireless Sensor Network; WSN) aus energetischer Sicht keine signifikanten Unterschiede vorhanden sind. Weiterhin bietet die RFID-Technologie (vgl. [Har2]) eine vielversprechende Möglichkeit Datenlogger möglichst lange zu betreiben. Aus diesem Grund wird für die folgende Betrachtung ein Funksensornetzwerk mit Bluetooth- oder einer vergleichbaren Technologie dem Einsatz eines äußerst energieeffizienten Datenloggers gegenübergestellt.

Aufgrund der oben gezeigten Systemeigenschaften stellt sich die Frage, in welchem Anwendungsfall der Einsatz von Funksensornetzwerken den Datenloggern (unabhängig von den zusätzlichen Möglichkeiten der Online-Datenerfassung) aus wirtschaftlichen Gründen vorzuziehen sind. In der folgenden Betrachtung werden hierzu die Investitionskosten mit einer Lebenszeit $T_{\text {lif } e}$ angesetzt und die jährlichen Kosten miteinander verglichen (wobei lediglich eine statische Investitionsrechnung angesetzt wird).

Diese Entscheidung wird hauptsächlich von der gewünschten Aktualität der Messdaten $\Delta T_{K o m m}$, der Anzahl der Messstellen $N$ sowie nicht zuletzt den Personalkosten $c_{P}$ beeinflusst. Für den Einsatz'von Datenloggern summieren sich hierbei die Investitionskosten von den Loggern $K_{M S}$, der Software und der Schnittstelle zum PC $K_{B A S E}$, sowie die Personalkosten $c_{P}$ für das Auslesen der Daten, wobei diese ebenfalls von der Anzahl der Messstellen sowie der mittleren Auslesezeit $t_{P}$ pro Messstelle abhängig sind: Der Batteriewechsel sei hierbei vernachlässigt und bereits in der mittleren Auslesezeit berücksichtigt.

$$
k_{D L}=\frac{N \cdot K_{M S}+K_{B A S E}}{T_{\text {lif } e}}+N \cdot\left(\frac{T_{\text {year }}}{\Delta T_{\text {Komm }}} \cdot t_{P} \cdot c_{P}\right)
$$

Im Funksensornetzwerk setzen sich die Investitionskosten ebenfalls aus den Kosten pro Messstelle $K_{M S}$, den Kosten für Basisstation und Software $K_{B A S E}$ sowie den Kosten für die Schaffung der Kommunikationsinfrastuktur $K_{I N F R A}$ (Annahme: pro $N_{I N F R A}$ Messstellen ein Repeater) zusammen. Hinzu kommen lediglich die Personalkosten, welche zum Wechsel der Batterien erforderlich sind. Die Dauer dieser Arbeit sei ebenfalls $t_{P}$, wobei die Energieversorgungskosten $K_{B a t t}$ zu berücksichtigen sind

$k_{W S N}=\frac{N \cdot\left(K_{M S}+\frac{K_{I N F R A}}{N_{I N F R A}}\right)+K_{\text {BASE }}}{T_{\text {lif e }}}+N \cdot\left(\frac{8760 h}{\Delta T_{\text {Batt }}} \cdot\left(t_{P} \cdot c_{P}+K_{\text {Batt }}\right)\right)$

Die Grundlagen für die angestellten Berechnungen sind in Tabelle 3 dargestellt.

\begin{tabular}{|l|c|c|c|c|c|c|}
\cline { 2 - 7 } \multicolumn{1}{c|}{} & \multicolumn{3}{c|}{ worst } & \multicolumn{1}{c|}{ DL } & \multicolumn{3}{c|}{ WSN } \\
\multicolumn{1}{c|}{} & 250 & 150 & 50 & 400 & 250 & 150 \\
\hline$K_{M S}$ & 300 & 100 & 0 & 4000 & 2500 & 1500 \\
\hline$K_{\text {BASE }}$ & - & - & - & 1 & 3 & 5 \\
\hline$N_{I N F R A}$ & - & - & - & 500 & 350 & 200 \\
\hline$K_{I N F R A}$ & - & - & - & 1 & 2 & 5 \\
\hline$\Delta T_{\text {Batt }}$ & - & - & - & 6 & 4 & 2 \\
\hline$K_{\text {Batt }}$ & 15 & 10 & 5 & 15 & 10 & 5 \\
\hline$t_{P}$ & 50 & 25 & 15 & 50 & 25 & 15 \\
\hline$c_{P}$ & 10 & 15 & 20 & 10 & 15 & 20 \\
\hline$T_{\text {lif } e}$ & 10 & & & best & worst \\
\hline
\end{tabular}

Tabelle 3: Vergleich der Kosten Drahtloses Sensornetz und Datenlogger 


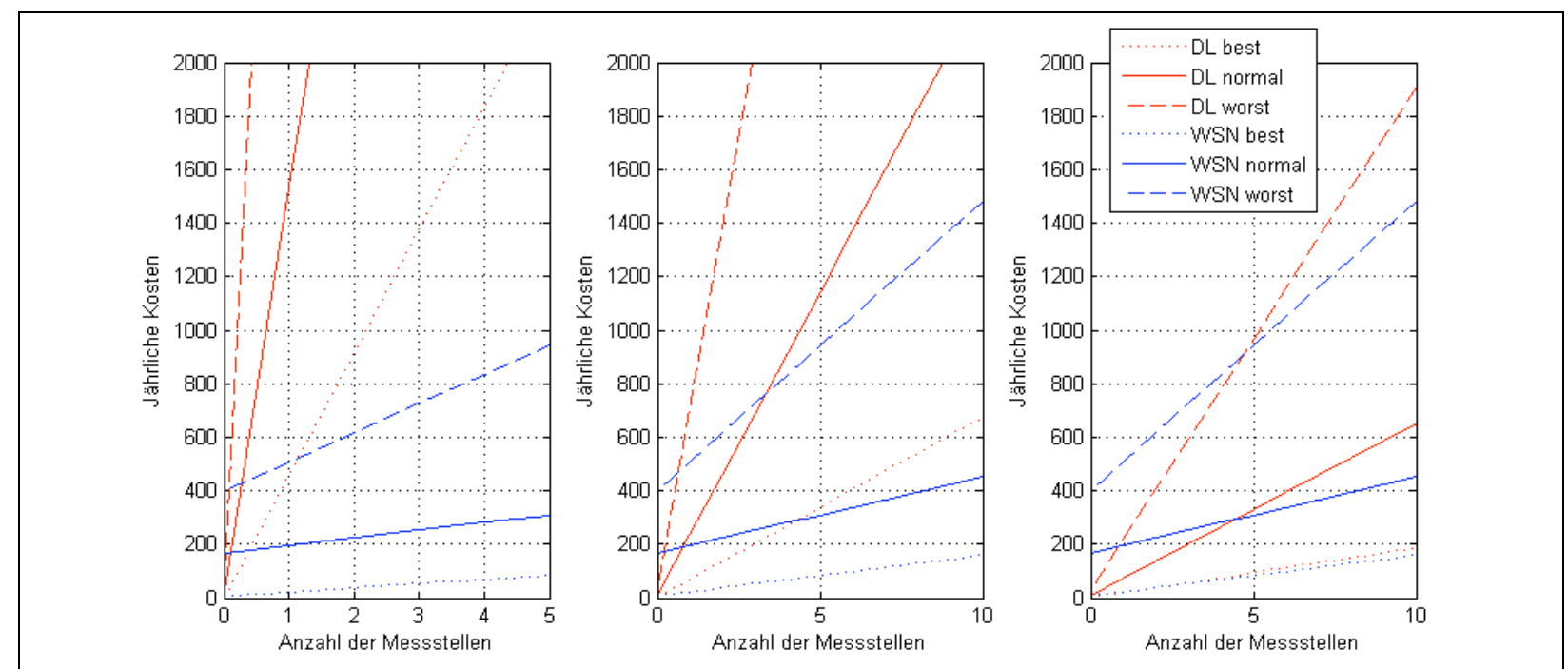

Abbildung 6: Vergleich der jährlichen Kosten für die Klimadatenerfassung im Bereich der präventiven Konservierung: Tagesaktualisierung (links), Wochenaktualisierung (mitte) und Monatsaktualisierung (rechts).

Der direkte Vergleich der Systeme ist in Abbildung 6 als jährliche Kosten für die Erfassung der Klimadaten bei unterschiedlicher Datenaktualität (links täglich, mitte wöchentlich, rechts monatlich). Erstaunlicherweise ist bei einer tagesaktuellen Messung bereits die Investition in ein drahtloses Sensornetzwerk sinnvoll, wenn mehr als eine Messstelle betrieben wird (Vergleich von „worst case“ WSN mit „best case“ DL). Bei einer wöchentlichen Aktualisierung sollte dies normalerweise mit einem konventionellen Sensornetzwerk der Fall sein, wenn die Messstellenanzahl etwa 5 übersteigt, bei einer monatlichen Erfassung kann keine robuste Aussage getroffen werden, da der Vergleich zu sehr von den tatsächlichen Systemanforderungen (Messgenauigkeit, Handling und Qualität des Produktes, usw.) und den damit verbundenen Kosten abhängig ist.

\section{Zusammenfassung und Ausblick}

Die Auswahl eines geeigneten Datenerfassungssystems ist auf Grund der vielfältigen Lösungsansätze lediglich durch klare Abgrenzung im Anwendungsgebiet und Definition der Anforderungen möglich. Der Umkehrschluss (die Einordnung der Systeme nach geeigneten Anwendungsgebieten) wird hier nochmals aufgeführt.

Aktive Datenlogger eignen sich zur zyklischen Datenübertragung und können durch die Auswahl der am besten geeigneten Übertragungsmöglichkeit energie- und kosteneffizient eingesetzt werden. Die Hauptkriterien stellen hierbei die Übertragungsentfernung sowie der Betriebszeitraum ohne Wartungsintervall dar. Bei niedrigen Datenmengen sowie kurzen Übertragungswegen eignen sich Systeme wie Infrarot oder besser noch RFID, um eine möglichst hohe Energieffizienz, bzw. einen möglichst langen Betriebszeitraum zu gewährleisten.

Sind höhere Datenmengen verlangt oder größere Übertragungsentfernungen zu überwinden, so kann zu Funkschnittstellen wie Bluetooth zurückgegriffen werden, deren höherer Energiegrundbedarf durch die höheren Übertragungsgeschwindigkeiten bei großen Datenmengen kompensiert wird.

Für einen möglichst langen Systembetrieb in der Applikation sollten Schnittstellen mit minimalem Energiebedarf, bezogen auf das Erfassungssystem, verwendet werden. Dies könnte eine USB-, RS-232oder eine drahtlose RFID Schnittstelle sein, wobei die zur Übertragung benötigte Energie jeweils vom auslesenden Gerät (PC, Lesegerät, etc.) gestellt wird. Eine zusammenfassende Gegenüberstellung ist in Abbildung 7 nochmals grafisch dargestellt. 


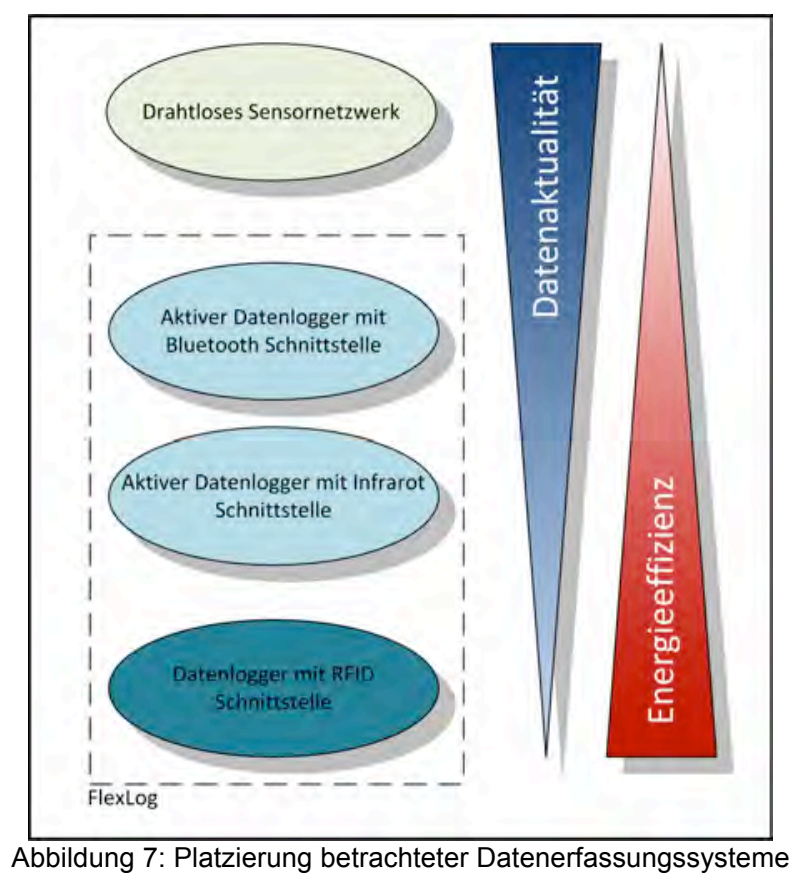

Energetische Betrachtungen wurden durchgeführt und eine Wirtschaftlichkeitsbetrachtung für einen speziellen Anwendungsfall vorgenommenen. Es konnte gezeigt werden, dass sich ein klarer Kostenvorteil für die Verwendung drahtloser Sensornetzwerke bei der Erfassung tages- und unter gewissen Umständen sogar wochenaktueller Daten besteht.

Um eine verlängerte Betriebsdauer von Datenloggersystemen zu gewährleisten wäre der Einsatz von Energy-Harvesting Systemen denkbar, potentielle Ansätze hierzu werden in Folgearbeiten am vorgestellten Flexlog-System untersucht.

\section{Literatur}

[ARN11] Arnold, C.; Lambeck, S.; Gieler, R.-P.: Klimastabilisierung bei beschränkten Eingriffsmöglichkeiten durch Handlungsempfehlungen; WTA-Schriftenreihe , Wissenschaftlich-Technische Arbeitsgemeinschaft zur Bauwerkserhaltung und Denkmalpflege, Heft 35, 2011, Fulda

[BLU11] Bluegiga: Product Brief, BLE112, 2011 http://www.bluegiga.com/files/bluegiga/Pub\%20files/BLE112_Product_Brief_140711_lores.pdf

[COS11] Costa, Guillermo N.; Arnaud, Alfredo: A low frequency RFID temperature data logger, Argentine School of Micro-Nanoelectronics Technology and Applications (EAMTA), 2011

[DIN09] DIN - Deutsches Institut für Normung e. V.: Erhaltung des kulturellen Erbes - Allgemeine Begriffe zur Erhaltung des kulturellen Erbes; Beuth-Verlag, Entwurf, DIN EN 15898, 2009

[FIN08] Finkenzeller, Klaus: RFID-Handbuch. Grundlagen und praktische Anwendungen von Transpondern, kontaktlosen Chipkarten und NFC, Hanser, 2008, S11 ff

[GES09] Gessler, R., Krause, T.: Wireless-Netzwerke für den Nahbereich, Vieweg+Teubner Verlag / GWV Fachverlage GmbH, Wiesbaden, 2009 S. 190-199

[HAR11a] Harasty, S., Arnold C., Björnsson, B., Michelmann, N.: Entwicklung von passiven und semipassiven RFID-Sensorsystemen für die Automatisierungstechnik, Automation 2011, S. $343 \mathrm{ff}$

[HAR11b] Harasty, S., Arnold C., Björnsson, B..: Klimadatenerfassung mit RFID, A\&D Kompendium 2011/2012, 2011, S.61 ff 
[HAR11c] Harasty, S.: Untersuchungen zur Entwicklung passiver RFID-Sensorsysteme auf 13,56 MHz Basis mit I² Schnittstelle, Bachelorthesis, Hochschule Fulda 2011

[IEE02] IEEE Project 802.15.1: Wireless Personal Area Network - Bluetooth, first Press release 2002

[ISO14] International Organization for Standardization (ISO), Identifikationskarten - Kontaktlose Chipkarten - Proximity-Karten, Norm 14443, 2010

[ISO15] International Organization for Standardization (ISO), "Identifikationskarten - Kontaktlose Chipkarten - "Vicinity"-Karten, Norm ISO 15693, 2010

[KNU04] Knutson, C., Brown, J.: IrDA Principles and Protocols. MCL Press, Salem UT 2004

[OPA11] OPAL Associates Holding AG: RFID Übersicht; http://www.rfid-loesungen.com, Aufgerufen am 15.12.2011

[SIG12] Bluetooth Special Interrest Group (SIG), A Look at the Basics of Bluetooth Wireless Technology, http://www.Bluetooth.com/Pages/Basics.aspx, Stand 05.01.12

[VIS09] Vishay, TFBS4650 data sheet, http://www.vishay.com/docs/84672/tfbs4650.pdf, 2009

[LIN08] Lina M. Pestana Leão de Brito, Laura M. Rodríguez Peralta, Filipe E. Sousa Santos, and Roberto P. Ramos Fernandes: Wireless Sensor Networks Applied to Museums' Environmental Monitoring; 4. International Conference on Wireless and Mobile Communications, Athen, Griechenland, 2008

[YON09] Yong-min, L.; Wu-Yi, L.: A Library's Environmental Monitoring System Based on Wireless Sensor Networks; International Conference on Networking and Digital Society, Cancun, Mexico, 2009 